\title{
GEOMETRY OF VORTICES AND DOMAIN WALLS
}

\author{
TOMASZ DOBROWOLSKI
}

Communicated by Ivaïlo M. Mladenov

\begin{abstract}
In this paper we consider relativistic models that contain in their spectra of solutions extended topological defects. We find the geometrical constrains that describe deformed vortices and domain walls of constant width. Analytical form of these solutions in co-moving coordinates is identical with analytical form of the appropriate static solutions in the laboratory Cartesian coordinates. The geometrical constrains presented here describe fully the shape and the evolution of the vortices and domain walls of constant width.
\end{abstract}

\section{Introduction}

In many branches of physics significant part of the physical phenomena are described by topological solitons of varied types. The most important, in the midst of topological defects, are extended topological configurations. There are two types of these configurations: vortices and domain walls.

The vortices appear in the description of many low energy systems. The most important examples of condensed matter systems that contain vortices are superconductors of second type where vortices have a form of magnetic flux tubes that puncture the bulk of the superconducting material [16]. In liquid crystals vortices have a form of optical fibres [8], [6]. Vortices of topological origin were also measured in superfluid Helium [11]. It seems that vortices may also play some role in the modern cosmology [22], [18], [14]. Finally, there is hypothesis that quarks in barions and mesons are connected by vortices that have a form of color flux tubes [7], [15], [8], [20].

On the other hand, domain walls are usually observed in ferromagnetic and ferroelectric materials.

The typical model that contain vortices is an Abelian Higgs model. This model describes a charged complex scalar field interacting with a vector potential (an electromagnetic potential)

$$
\mathcal{L}=-\frac{1}{4} F_{\mu \nu} F^{\mu \nu}+\left(D^{\mu} \phi\right)^{*}\left(D_{\mu} \phi\right)+\frac{1}{4} \lambda\left(\phi \phi^{*}-a\right)^{2}
$$


where $D_{\mu} \phi=\partial_{\mu} \phi+\mathrm{i} q A_{\mu} \phi$ and $F_{\mu \nu}=\partial_{\mu} A_{\nu}-\partial_{\nu} A_{\mu}$ is the electromagnetic field strength tensor. The equations of motion in this system are

$$
D_{\mu} D^{\mu} \phi+\frac{1}{2} \lambda \phi\left(\phi \phi^{*}-a\right)=0
$$

and

$$
\partial^{\mu} F_{\mu \nu}=\mathrm{i} q\left(\phi^{*} D_{\nu} \phi-\phi D_{\nu} \phi^{*}\right) .
$$

It was proved that in this model there exists cylindrically symmetric static solutions (with finite energy per unit length) of the form [1], [19]

$$
\begin{aligned}
\phi & =\phi_{V}(r, \varphi)=F(r) \mathrm{e}^{\mathrm{i} n \varphi} \\
A_{\mu} & =(0,-y A(r), x A(r), 0)
\end{aligned}
$$

where $F$ and $A$ are well defined functions of radial variable. The typical profile of the function $F$ is presented in Figure 1. In the same figure one can find the " $\mathrm{z}$ " component of the magnetic field. Unfortunately, the analytical form of functions $F$ and $A$ for arbitrary parameters of the model are not known. The particular form of the solution (2) follows from general considerations. First, the scalar field disappears on "Z" axis and therefore $F(r) \rightarrow 0$ for $r \rightarrow 0$. This condition guarantees non-singularity of the scalar field. Second, uniqueness of the scalar field is guaranteed by the choice of the phase factor $n \varphi$. On the other hand the energy per unit length is finite if $F(r) \rightarrow \sqrt{a}$ for $r \rightarrow \infty$ and vector potential is a pure gauge field. The equation (2) for $r \rightarrow \infty$ can be understood as a mapping from a circle located at spatial infinity $\mathbb{S}_{r \rightarrow \infty}^{1}$ to a vacuum manifold $\mathbb{S}_{\sqrt{a}}^{1}$. The index of this mapping $n$ indicates how many times the second circle is surrounded if the first one is surrounded only once. This index (called a winding number or a topological charge) divides vortex solutions on non equivalent classes. Each class consists of the static solution of given topological charge $n$ and other excited vortices characterized by the same winding number. These excited solutions, in general depend on other variables, are non-static and does not posses cylindrical symmetry

$$
\phi=\phi_{V}(r, \varphi)=F(r, \varphi, z, t) \mathrm{e}^{\mathrm{i} n \varphi} .
$$

The solutions of this type are studied in the framework of the perturbation scheme [3] (a similar studies were performed in a case of domain walls [3], [4]). The classes are separated from each other by infinite energy barrier and therefore the solutions (that minimalize the energy per unit length) are stable [17]. In the present paper we reduce the dynamics of the vortex to the dynamics of the line of zeros of the scalar field. This line can be considered as a string that represents a vortex 


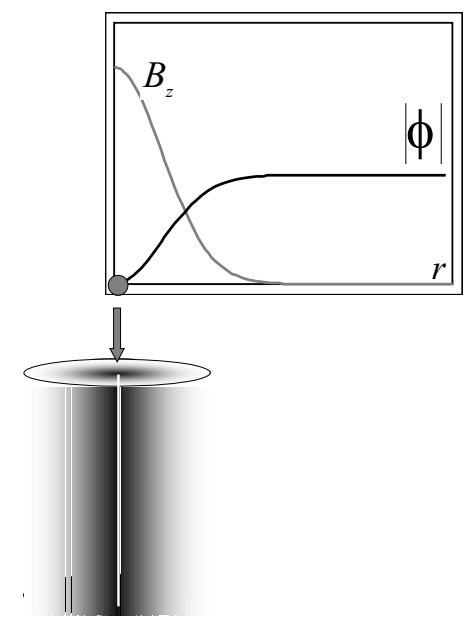

Figure 1. The scalar field drops to zero on the " $Z$ " axis. The third component of the magnetic field is substantially different from zero in the vicinity of the "Z" axis.

[12], [13]. In order to have direct correspondence between vortices and strings we restrict our considerations only to vortices of constant width. The same approach can be used in order to describe the domain walls. The only difference is that in case of domain walls the zeros of the scalar field lie on the two dimensional surface.

\section{Co-moving Coordinates}

\subsection{Coordinates in the Vicinity of the String}

In the previous section we identified the vortex with the string. In case of the straight string its points can be indicated, for example, by " $\mathrm{z}$ " variable. If the string is curved then we use the space-like parameter $\sigma$. During its evolution the string leaves a trail in the space-time that has a form of the $1+1$ dimensional manifold $\Sigma$ called the word-sheet. For convenience we introduce more compact notation: $\left(\sigma^{a}\right)=\left(\sigma^{0}, \sigma^{3}\right)=(\tau, \sigma)$. The position (in the Cartesian laboratory frame) of the point on the word-sheet can be uniquely defined by the radius vector field $X^{\mu}\left(\sigma^{a}\right)$. Near the world-sheet one can introduce the co-moving coordinates $\left(\zeta^{\alpha}\right)=\left(\sigma^{a}, \rho^{i}\right)$. The additional coordinates $\left(\rho^{i}\right)=\left(\rho^{1}, \rho^{2}\right)$ are normal to the world-sheet and they describe how far is a point from the surface $\Sigma$. There exists 
simple relation between co-moving and laboratory Cartesian coordinates

$$
x^{\mu}=X^{\mu}\left(\sigma^{a}\right)+\rho^{i} n_{i}^{\mu}\left(\sigma^{a}\right)
$$

where $n_{i}^{\mu}\left(\sigma^{a}\right)$ are two vectors $(i=1,2)$ normal to the surface $\Sigma$ at each point $X^{\mu}\left(\sigma^{a}\right)$. Additionally we assume that they are orthonormal and space-like fourvectors

$$
n_{i}^{\mu} n_{j \mu}=-\delta_{i j}, \quad n_{i \mu} X_{, a}^{\mu}=0 .
$$

The minus sign in the first relation of the equation (6) is consistent with metric convention $(+,-,-,-)$. The Cartesian coordinates in the laboratory frame are denoted in standard way, i.e., $x^{\mu}$. In the paper we also use the metric induced on the surface $\Sigma$

$$
X_{, a}^{\mu} X_{\mu, b} \equiv g_{a b}
$$

where $X_{, a}^{\mu}$ is tangent vector to $\Sigma$ in the direction of the $\sigma^{a}$ variable. The embedding of the world-surface is described by the extrinsic curvature coefficients $K_{a b}^{i}$ and the torsion coefficients $\omega_{a}$ that follows from the Gauss-Weingarten formulas

$$
\begin{aligned}
\partial_{a} X_{, b}^{\mu} & =X_{, a b}^{\mu}=\Gamma_{a b}^{c} X_{, c}^{\mu}+K_{a b}^{i} n_{i}^{\mu} \\
\partial_{a} n_{i}^{\mu} & =n_{i, a}^{\mu}=K_{a b}^{i} g^{b c} X_{, c}^{\mu}+\epsilon^{i k} \omega_{a} n_{k}^{\mu}
\end{aligned}
$$

where $\epsilon^{i j}$ is Levi-Civita symbol. In the description of the string we have additionally a freedom of rotation of the normal frame $n_{i}^{\mu}$ to the word-sheet. This symmetry is taken into account by introducing the covariant derivatives with respect to $\sigma^{a}$ variables

$$
\hat{\partial}_{a} f \equiv \partial_{a} f-\omega_{a} \partial_{\varphi} f
$$

\subsection{Coordinates in the Neighborhood of the Domain Wall}

A similar construction can be performed in the case of the domain wall. In this particular case the surface is parameterized by two space-like variables $\left(\sigma^{1}, \sigma^{2}\right)$. During its evolution the domain wall leaves in the space-time a trail in the form of $2+1$ dimensional manifold $\tilde{\Sigma}$ parameterized by parameters $\left(\sigma^{a}\right)=\left(\sigma^{0}, \sigma^{1}, \sigma^{2}\right)=$ $\left(\tau, \sigma^{1}, \sigma^{2}\right)$. This manifold is called word-hypersurface.

Similarly like in the string case, near the hypersurface $\tilde{\Sigma}$ we introduce the comoving coordinates $\left(\zeta^{\alpha}\right)=\left(\sigma^{a}, \xi\right)$

$$
x^{\mu}=X^{\mu}\left(\sigma^{a}\right)+\xi n^{\mu}\left(\sigma^{a}\right)
$$


where the vector $n^{\mu}$ is normal to the membrane and $\xi$ is a coordinate in the direction of the vector $n^{\mu}$. By definition the space-like four-vector $n^{\mu}$ is normalized to unity and is orthogonal to the vectors $X_{, a}^{\mu}$ - tangent to the world-hypersurface $\tilde{\Sigma}$

$$
n^{\mu} n_{\mu}=-1, \quad n_{\mu} X_{, a}^{\mu}=0 .
$$

The minus sign in the first relation of the equation (8) is consistent with metric convention $(+,-,-,-)$. The embedding of the world-hypersurface is described by the extrinsic curvature coefficients $K_{a b}$ and they follows from the Gauss-Weingarten formulas

$$
\partial_{a} X_{, b}^{\mu}=X_{, a b}^{\mu}=\Gamma_{a b}^{c} X_{, c}^{\mu}+K_{a b} n^{\mu}, \quad \partial_{a} n^{\mu}=n_{, a}^{\mu}=K_{a}^{c} X_{, c}^{\mu} .
$$

In this description we have only one normal dimension and therefore we have no torsion and no complication connected to rotation of normal frame.

\section{Method of Construction of the Cured Vortex or Domain Wall}

The method used in order to find the geometry of constant width vortices and domain walls can be explained on a simple example. Let us consider some partial differential equation

$$
\hat{O}_{x, y} \phi(x, y)=0
$$

Let us also presume that we know exact (although particular) solution of this equation

$$
\phi_{0}(x, y)
$$

This equation can be transformed into arbitrary coordinates

$$
x^{\prime}=f(x, y), \quad y^{\prime}=g(x, y) .
$$

In these coordinates the equation usually changes its form to

$$
\hat{O}_{x^{\prime}, y^{\prime}} \phi\left(x^{\prime}, y^{\prime}\right)=0 \text {. }
$$

The solution (9) in the new coordinates usually also changes its form

$$
\tilde{\phi}_{0}\left(x^{\prime}, y^{\prime}\right) \text {. }
$$

A simple example of such equation is

$$
\left(\partial_{x}^{2}+\partial_{y}\right) \phi(x, y)=0
$$


with particular solution of the form

$$
\phi_{0}(x, y)=\mathrm{e}^{x-y} .
$$

After transformation

$$
x^{\prime}=x, \quad y^{\prime}=x+y
$$

the equation becomes

$$
\left(\partial_{x^{\prime}}^{2}+2 \partial_{x^{\prime}} \partial_{y^{\prime}}+\partial_{y^{\prime}}^{2}+\partial_{y^{\prime}}\right) \phi\left(x^{\prime}, y^{\prime}\right)=0
$$

and the solution change its form

$$
\tilde{\phi}_{0}\left(x^{\prime}, y^{\prime}\right)=\mathrm{e}^{2 x^{\prime}-y^{\prime}} .
$$

Let us notice that the analytical form of the solution differs from the solution given by the formula (11). On the other hand one can check that the function $\phi_{0}\left(x^{\prime}, y^{\prime}\right)$ which has identical analytical form as the solution of the original equation but written in new coordinates $\left(x^{\prime}, y^{\prime}\right)$

$$
\phi_{0}\left(x^{\prime}, y^{\prime}\right)=\mathrm{e}^{x^{\prime}-y^{\prime}}
$$

is not a solution of the equation (12) in these coordinates.

In fact we post the inverse problem: we will look for transformations (10) that does not change the analytical form of the solution (9). Moreover, we will concentrate only on transformations to co-moving coordinates. The special status of these coordinates follows from the fact that they directly describe the shape and the evolution of the vortex (or domain wall).

Actually, if we have defined the co-moving coordinates we can make the step back (using relations (5) or (7)) and find analytical form of the solutions in the laboratory frame or use the equations that define the geometry of the surface $\Sigma$ (or hypersurface $\tilde{\Sigma}$ ) in order to define perturbational scheme for vortices with changeable with.

\section{Evolution of the Constant Width Defects}

\subsection{Geometry of the Vortex World Surface}

The simplest models that contain vortex solutions are defined by the Lagrangian density

$$
\mathcal{L}=\partial_{\mu} \phi \partial^{\mu} \phi^{*}-V\left(\phi^{*} \phi\right)
$$


where $\phi$ is a complex scalar field. An example of this kind of model is the Goldstone model defined by the potential $V\left(\phi^{*} \phi\right)=\frac{\lambda}{4}\left(\phi \phi^{*}-a\right)^{2}$, where $\lambda$ and $a$ are positive constants. The corresponding Euler - Lagrange equation have the form

$$
\partial_{\mu} \partial^{\mu} \phi+\frac{\delta V}{\delta \phi^{*}}=0
$$

We presume that in the considered model the equation of motion has static, cylindrically symmetric vortex solutions (2). Although, analytical form of the function $F$ is not known explicitly, we presume that it is defined by the expansion in the radial variable. The equation of motion in the co-moving coordinates has the form

$$
\begin{aligned}
\widehat{\partial}_{a}\left(\sqrt{-g} \mathcal{G} G^{a b} \widehat{\partial}_{b} \phi\right)-\partial_{r}\left(\sqrt{-g} \mathcal{G} \partial_{r} \phi\right)-\frac{1}{r} \sqrt{-g} \mathcal{G} \partial_{r} \phi \\
-\frac{1}{r^{2}} \partial_{\varphi}\left(\sqrt{-g} \mathcal{G} \partial_{\varphi} \phi\right)+\sqrt{-g} \mathcal{G} \frac{\delta V}{\delta \phi^{*}}=0
\end{aligned}
$$

where $r=\sqrt{\left(\rho^{1}\right)^{2}+\left(\rho^{2}\right)^{2}}$. Here $g=\operatorname{det}\left[g_{a b}\right]$ is determinant of the metric induced on the world-sheet

$$
\mathcal{G}=1+r K_{a}^{r a}+\frac{1}{2} r^{2}\left[K_{a}^{r a} K_{b}^{r b}-K_{b}^{r a} K_{a}^{r b}\right]
$$

and

$$
K_{a b}^{r}=K_{a b}^{1} \cos \varphi+K_{a b}^{2} \sin \varphi .
$$

In the equation (14) we also use the components of the inverse metric tensor in the co-moving coordinates

$$
G^{a b}=\mathcal{G}^{-2}\left[g^{a b}\left(1+r K_{c}^{r c}\right)^{2}-2 r K^{r a b}\left(1+r K_{c}^{r c}\right)+r^{2} K_{c}^{r a} K^{r b c}\right] .
$$

We assume that equation (14) in the co-moving coordinates posses vortex solution with the analytical form identical to the form defined by the Euler-Lagrange equation in the laboratory frame, i.e.,

$$
-\partial_{r}^{2} \phi_{V}-\frac{1}{r} \partial_{r} \phi_{V}-\frac{1}{r^{2}} \partial_{\varphi}^{2} \phi_{V}+\left.\frac{\delta V}{\delta \phi^{*}}\right|_{\phi=\phi_{V}}=0
$$

where $r=\sqrt{x^{2}+y^{2}}$ and $\phi_{V}(r, \varphi)=F(r) \mathrm{e}^{\mathrm{i} n \varphi}$. In particular the last equation (15) defines expansion of function $F$ in the radial variable $r$. If we use an information contained in the equation (15) then we can remove the nonlinear term from equation of motion in co-moving coordinates (14) and separate this complex equation on imaginary and real part

$$
\frac{1}{\sqrt{-g}} \partial_{a}\left(\sqrt{-g} \mathcal{G} G^{a b} \omega_{b}\right)-\partial_{\varphi}\left(\mathcal{G} G^{a b}\right) \omega_{a} \omega_{b}+\frac{1}{r^{2}} \partial_{\varphi} \mathcal{G}=0
$$




$$
n^{2} \mathcal{G} G^{a b} \omega_{a} \omega_{b} F+\partial_{r} \mathcal{G} \partial_{r} F=0 .
$$

Next, we expand the equations (16), (17) in the radial variable and obtain from the subsequent orders of this expansion the finite number of conditions that define the geometry of the world-sheet of the string

$$
\omega_{a}=0, \quad K_{a}^{i a}=0, \quad K_{a}^{i b} K_{b}^{j a}=0, \quad i, j=1,2 .
$$

The above conditions describe the Nambu-Goto string with some additional constrains. First, let us notice that the number of independent conditions obtained in this way is finite - although the number of orders of expansion is unfine. Second, although the above constrains (18) are quite restrictive they allow existence of nontrivial solutions. An example of such solution is a surface of the form

$$
X^{\mu}=\left[\tau, \psi_{1}(\sigma \pm \tau), \psi_{2}(\sigma \pm \tau), \sigma\right]
$$

where $\psi_{1}(\sigma \pm \tau)$ and $\psi_{2}(\sigma \pm \tau)$ are almost arbitrary functions of the light-cone coordinates. In fact we presumed that $\psi_{i}$ are three times integrable functions of its arguments (here $c=1$ ). Having the solution (19) one can use relation (5) in order to transform the vortex solution $\phi\left(\rho^{1}, \rho^{2}\right)$ into laboratory coordinates. The final outcome of this calculus is a solution in the form of Vachaspati wave [21]

$$
\phi_{V}\left(x-\psi_{1}(z \pm t), y-\psi_{2}(z \pm t)\right) .
$$

This solution is represented by the threefold in Figure 2 (as a deformed worldsheet, as an evolved string and as an evolved vortex). Let us notice that for the obtained string conditions (18) the co-moving coordinates are defined globally. The last observation is visible from the determinant of the metric in the co-moving coordinates

$$
\sqrt{-G}=\sqrt{-g}\left[1+r K_{a}^{r a}+\frac{1}{2} r^{2}\left[K_{a}^{r a} K_{b}^{r b}-K_{b}^{r a} K_{a}^{r b}\right]=\sqrt{-g} \neq 0 .\right.
$$

During considerations of the vortex evolution we assumed also that the worldsurface is integrable and therefore satisfies Peterson-Codazzi, Ricci and Gauss integrability conditions. The last of these conditions establishes explicit connection between external and internal geometry of the manifold $\Sigma$ described by the components of the Riemann curvature tensor

$$
R_{a b c d}=K_{a d}^{i} K_{b c}^{i}-K_{a c}^{i} K_{b d}^{i} .
$$

Immediate consequence of the equations (18) and the Gauss integrability condition is triviality of the internal geometry of the world-sheet of the considered string. 

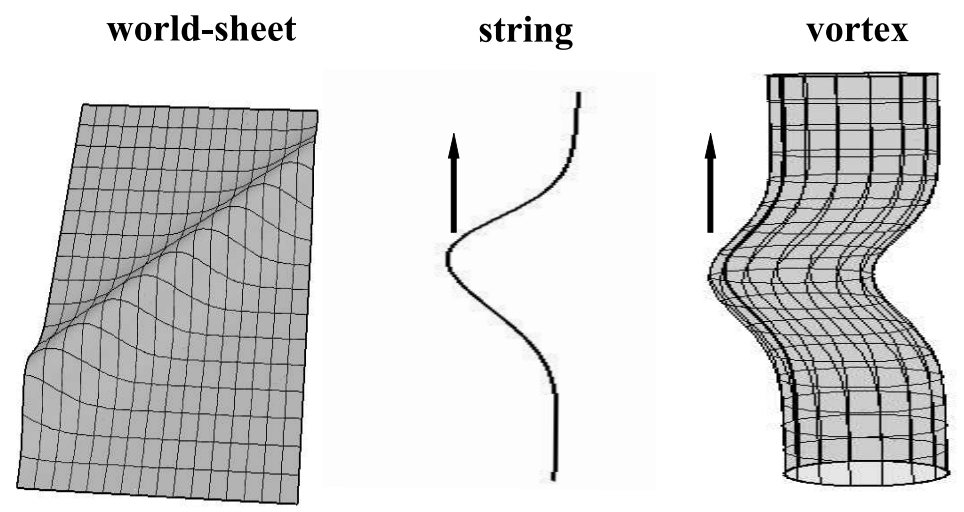

Figure 2. The solution (19) presented i the form of the world-sheet, line of zeros of the scalar field and a vortex.

One can see that the Riemann curvature scalar is zero for surfaces defined by the string equations

$$
R=K_{a}^{i b} K_{b}^{i a}-K_{a}^{i a} K_{b}^{i b}=0 .
$$

This result means that the world-surface of the vortex has geometry similar to the geometry of the sheet of paper embedded nontrivially in the three dimensional Euclidean space. Finally, it is worth to mention that identical set of equations, i.e., (18) was obtained for the Abelian Higgs model (1), however in this case the result was obtained under assumption of triviality of the torsion, i.e., $\omega_{a}=\partial_{a} \chi$ (where $\chi$ is an arbitrary function).

\subsection{Geometry of the Domain Wall World Hyper-surface}

The simplest models that contain in the spectra of solutions domain walls are built of real scalar field $\phi$

$$
\mathcal{L}=\frac{1}{2} \partial^{\mu} \phi \partial_{\mu} \phi-V(\phi)
$$

In the typical example of the $\phi^{4}$ model the domain walls are given by hyperbolic tangent function. The corresponding Euler - Lagrange equation have the form

$$
\partial_{\mu} \partial^{\mu} \phi+\frac{\delta V}{\delta \phi}=0
$$

The same equation in the co-moving coordinates reads

$$
\frac{1}{\sqrt{-G}} \partial_{a}\left(\sqrt{-G} G^{a b} \partial_{b} \phi\right)-\frac{1}{\sqrt{-G}} \partial_{\xi}\left(\sqrt{-G} \partial_{\xi} \phi\right)+\frac{\delta V}{\delta \phi}=0
$$


where components of the inverse metric in the co-moving coordinates have the form

$$
G^{a b}=\left(S^{-1}\right)_{c}^{a}\left(S^{-1}\right)^{b c}
$$

and $\left(S^{-1}\right)^{a b}$ are inverse to $S_{a b} \equiv g_{a b}+\xi K_{a b}$. Moreover $G$ is determinant of the metric tensor in the co-moving coordinates

$$
\sqrt{-G}=\sqrt{g} \mathcal{G}
$$

where

$$
\begin{aligned}
\mathcal{G}=1+\xi K_{a}^{a}+\frac{1}{2} \xi^{2}\left[K_{a}^{a} K_{b}^{b}\right. & \left.-K_{b}^{a} K_{a}^{b}\right] \\
& +\frac{1}{6} \xi^{3}\left[K_{a}^{a} K_{b}^{b} K_{c}^{c}-3 K_{a}^{a} K_{c}^{b} K_{b}^{c}+2 K_{b}^{a} K_{c}^{b} K_{a}^{c}\right] .
\end{aligned}
$$

We assume that the equation of motion have static solution in the form of domain wall $\phi_{W}=\phi_{W}(z)$. In this article we look for possible deformations of the domain wall solution and therefore we assume that the analytical form of the function $\phi=\phi_{W}(\xi)$ is identical with the solution of the equation (20), i.e.,

$$
-\partial_{\xi}^{2} \phi_{W}+\left.\frac{\delta V}{\delta \phi}\right|_{\phi=\phi_{W}}=0 .
$$

The natural consequence of the adopted form of the field $\phi$ is lack of its dependence on the variables $\sigma^{a}$, i.e., $\partial_{a} \phi_{W}=0$. The equation of motion (21) for function $\phi_{W}$ reduces substantially

$$
-\frac{1}{\mathcal{G}} \partial_{\xi}(\mathcal{G}) \partial_{\xi} \phi_{W}=0
$$

In the last equation the function $\phi_{W}$ has fixed shape and the only unknown are coefficients of the external curvature $K_{a b}$. In the next step we expand equation (23) with respect to the normal coordinate $\xi$

$$
\left[-K_{a}^{a}+\xi K_{b}^{a} K_{a}^{b}-\xi^{2} K_{b}^{a} K_{c}^{b} K_{a}^{c}+\ldots\right] \partial_{\xi} \phi_{W}(\xi)=0 .
$$

Moreover, expanding $\phi_{W}(\xi)$ and then separating coefficients of subsequent orders of expansion of the whole equation (24) we obtain the finite set of independent conditions

$$
K_{a}^{a}=0, \quad K_{a}^{b} K_{b}^{a}=0, \quad K_{b}^{a} K_{c}^{b} K_{a}^{c}=0 .
$$

In addition, let us notice that from equation (22) it follows that conditions (25) guarantee global existence of the used by us curvilinear coordinates

$$
\sqrt{-G}=\sqrt{g} \neq 0 .
$$


Summing up, we have found that the shape of the domain wall and its evolution is exactly and globally described by the set of geometrical equations (25). Similarly, like in the case of the vortex solution we can indicate nontrivial solutions of the membrane equations (25)

$$
X^{\mu}=\left[\tau, \sigma^{1}, \sigma^{2}, \psi\left(\tau-v^{1} \sigma^{1}-v^{2} \sigma^{2}\right)\right]
$$

where $c=1$ and $\psi$ is three times integrable function of its arguments. We also assume that constants $v^{1}$ and $v^{2}$ are not independent and they satisfy relation $\left(v^{1}\right)^{2}+\left(v^{2}\right)^{2}=1$. The proposed hypersurface describes deformed membrane with a deformation of arbitrary shape $\psi$ propagating along the membrane with the speed of light.

\section{Acknowledgements}

I would like to thank Ivaïlo Mladenov and other organizers of the conference for hospitality during my stay in Varna.

\section{References}

[1] Abrikosov A., On the Magnetic Properties of Superconductors of the Second Group, Sov. Phys. JETP 5 (1957) 1174-1182.

[2] Arodź H., On Expansion in the Width for Domain Walls, Nucl. Phys. B 450 (1995) 174-188.

[3] Arodź H., Expansion in the Width: the Case of Vortices, Nucl. Phys. B 450 (1995) 189-208.

[4] Arodź H., Expansion in the Width and Collective Dynamics of a Domain Wall, Nucl. Phys. B 509 (1998) 273-293.

[5] Baker M., Ball J. and Zachariasen F., Chiral-Symmetry Breaking in Dual QCD, Phys. Rev. D 38 (1988) 1926-1933.

[6] Bowick M., Chandar L., Schiff E. and Srivastava A., The Cosmological Kibble Mechanism in the Laboratory: String Formation in Liquid Crystals, Science 263 (1994) 943-945.

[7] Brout R., Englert F. and Fishler W., Magnetic Confinement in Non-Abelian Gauge Field Theory, Phys. Rev. Lett. 36 (1976) 649-652.

[8] Chandrasekhar S. and Ranganath G., The Structure and Energetics of Defects in Liquid Crystals, Adv. Phys. 35 (1986) 507-596. 
[9] Dobrowolski T., Construction of Curved Domain Walls, Phys. Rev. E 77 (2008) 056608.

[10] Dobrowolski T., Construction of Curved Global Vortex, Ann. of Phys. 324 (2009) 2473-2489.

[11] Donnelly R., Quantized Vortices in Helium II, Cambridge Univ. Press, Cambridge, 1991.

[12] Förster D., Dynamics of Relativistic Vortex Lines and Their Relation to Dual Theory, Nucl. Phys. B 81 (1974) 84-92.

[13] Gervais J. and Sakita B., Quantized Relativistic String as a Strong Coupling Limit of the Higgs Model, Nucl. Phys. B 91 (1975) 301-316.

[14] Hindmarsh M. and Kibble T., Cosmic Strings, Rep. Prog. Phys. 58 (1995) 477-562.

[15] t'Hooft G., A Property of Electric and Magnetic Flux in Non-Abelian Gauge Theories, Nucl. Phys. B 153 (1979) 141-160.

[16] Huebener R., Magnetic Flux Structures in Superconductors, Springer, Berlin, 1979.

[17] Jaffe A. and Taubes C., Vortices and Monopoles, Birkhauser, Boston, 1980.

[18] Kolb E. and Turner M., The Early Universe, Addison-Wesley, Redwood, 1990.

[19] Nielsen H. and Olesen P., Vortex-line Models for Dual Strings, Nucl. Phys. B 61 (1973) 45-61.

[20] Olson C., Olsson M. and Williams K., Relativistic Flux Tubes and Potential Models QCD, Phys. Rev. D 45 (1992) 4307-4311.

[21] Vachaspati T., Travelling Waves on Domain Walls and Cosmic Strings, Phys. Lett. B 238 (1990) 41-44.

[22] Vilenkin A. and Shellard E., Cosmic Strings and Other Topological Defects, Cambridge Univ. Press, Cambridge, 1994.

Tomasz Dobrowolski

Institute of Physics UP

30-084 Cracow, POLAND

E-mail address: dobrow@up.krakow.pl 